suchen, ob auch an diesex eine Hyperplasie zu besbachten ist, welche anf die Ernährung des Eies von Einfluss sein könnte.

Die Gelegenheit zu dieser Untersuchung wird sich bald wieder Jemandem darbieten, denn die Endometritis decidualis polyposa ist gewiss nicht so selten, wie man aus der kleinen Zahl der bisher veröffentlichten Beobachtungen glauben möchte.

Mitte Juni d. J. konnte ich der Gesellschaft firr Geburtshülfe zu Leipzig bereits vier derartige Präparate vorlegen. Seitdem abortirte Frau B. zum zweiten Male, Auch diese Decidua zeigte meluere langgestielte Polypen. Somit habe ich an fünf Eiern hinter einander behandelter Aborte die Deciduawucherungen gefunden.

22. Juli 1876.

\title{
Zur Anteflexionsfrage.
}

\author{
Vou. \\ P. Mũller
}

in Bern.

Die Erörterungen über die Therapie der Uterustexionen, welche in den letzten Jahren die Gynäkologen beschäftigten, scheinen jetzt der erneuten Besprechung der Aetiologie dieser Formveränderung der Gebärmutter Platz machen zu wollen. Die Ansichten über die Vorwärtsbeugung, welche B. Schultze in diesem Archive) äusserte, dürften dazu den Anstoss gegeben haben. Schom ist von competenter Seite ${ }^{2}$ ) eine Aeusserung zur Richtigstellung der Schnltze'schen Anschauung erfolgt; auch ich darf mir hier exlauben, letztere an der Hand meiner Erfahrungen ganz in Kürze zu prüfen.

Die Ansicht von B. Schultze läuft bekanntlich darauf hinaus, dass die von ihm sogenannte pathologische Anteflexion am häufigsten durch eine Schrumpfung und Verkürzung der Douglas'schen Falten in Folge einer eigenthümlichen Parametritis posterior entstehe und zwar in der Weise, dass die Cervix an die hintere Beckenwand fixirt und der Uteruskörper dann durch intraabdominellen Druck nach abwärts gedrängt werde. Sterilität und Dysmenorrhve, die Begleiterinnen der Anteflexionen, seien weniger durch die Formveränderung des Gebärorganes als durch entzïndliche Affection in und um den

1) Band VIII, S. 134.

2) K. Schröder, Dieses Arehiv, Band IX, S. 68. 
Uterus bedingt. Diese Ansicht, so plausibel dieselbe auch für den ersten Augenblick sein mag, ist jedoch keineswegs neu. Hat doch E. Martin in seinem Werke über die Neigungen und Beugungen der Gebärmutter einen Theil der Anteflexion auf eine Fixirung des Cervix an die hintere Beckenwand zurückgeführt; nur lässt Martin ausser einer Schrumpfung der Ligamenta sacro-uterina auch eine intraparietale Anlöthung des Uterus in Folge von perimetrischen Exgudaten zu. E. Martin hat diesen Zustand, was Entstehung, Verlauf u. s. w. anlangt, so trefflich geschildert, dass die Schultze' schen Auseinandersetzungen doch nur als weitere Ausführangen betrachtet werden können. Neu ist an der Schultze'schen Publication eigentlich nur - wenn man sich auf das Wesentliche beschränkt - die Behauptung, dass fast alle Flexionen und nicht blos ein kleinerer Bruchtheil, einerlei ob sie sich bei Franen, die geboren haben oder bei Sterilen vorfinden, auf die angegebene Weise entstehen. Es fragt sich nun, wie es sich mit dieser Fixirung des Uterus an die hintere Beckenwand und besonders ihrer Häufigkeit verhält.

Gewiss kann es nicht bestritten werden, dass nach puerperalen Entziindungen der Uterus durch Schrumpfung der Ligamenta sacrouterina und durch Pseudomembranen extramedian gestellt und der Uteruskörper im schlaffen Zustande dadurch in Flexionsstellung gebracht werden kann; eben so sicher ist es, dass auch der jungfräuliche Uterus durch intrauterine Exsudate und Extravasate - deren genauere Bekanntschaft wir ja in letater Zeit gemacht haben respective deren Residuen eine gleiche Formveränderung eingehen kann. Darf man hierin mit B. Schultze übereinstimmen, so muss doch entschieden die Behauptung desselben, als wäre die abnorme Fixation fast die einzige Ursache der Anteflexionen, zurückgewiesen werden. Den Nachweis zu liefern, dass die Anteflexionen, welche entweder direct oder indirect nach einem Wochenbette entstehen, nicht immer durch eine puerperale Entzündung hinter dem Uterus vermittelt werden, möchte ich Anderen ïberlassen; hier möchte ich nur nachweisen, wie wenig auch für die Mehrzahl der Anteflexionen von Nulliparen diese Erklärungsweise in ihrer Ausschliesslichkeit passt. Das Material, auf welches ich mich stützen kann, ist ein ziemlich reichliches; werden doch jetzt junge Mädchen, bei denen die ersten Menstruationen mit Besehwerden verlaufen sind, sehr häufig dem Arzte zugeführt; suchen doch jetzt junge Frauen, wenn nicht schon im ersten Jahre der Ehe Conception eintritt, Hülfe bei dem Specialisten. Gerade in diesen Fällen stösst man sehr häufig auf Anteflexionen, welche hier bei intaeten oder doch wenig derangirten Genitalien zur Prüfung der Schultze'schen Ansicht sehr geeignet sind. Faälle von Sterilität, welche ja sehr häufig die Folge von Anteflexionen ist, habe ich zu Dutzenden mit genauer Notirung der anamnetischen Momente, Untersuchungsbefunde, Zeichnungen u. s. w. gesammelt. Da ich in Sterilitätsfallen ein grosses Gewicht auf etwaige Dislocation der Ovarien lege, so war ich stets in der Lage, meine Aufmerksamkeit gerade jener Gegend zuzuwenden, in der sich ja der von Schultze so sehr betonte Entziindungsprocess abspielt. Seit 
der Schultze'schen Publication haben sich mir die Fälle sehr vermehrt, an denen ich die Ansicht desselben direct prüfen konnte; ich muss jedoch gestehen, dieselben sprechen keineswegs für die Richtigkeit der Letzteren.

Was vor Allem die Ursachen anlangt, welche die fragliche Parametritis posterior hervorrufen sollen, so lauten dariber die Angaben ron Schultze ziemlich unbestimmt; es machen seine Bemerkungen hierüber den Eindruck, als ob in dieser Beziehung wenig Sicheres zu eruiren wäre. Auch ich konnte in der Mehrzahl der Fälle keine derartigen Momente ermitteln, wie sie von Schultze angefithrt werden. So hochgradige masturbatorische Reizungen, die doch nur durch eine fortgeleitete Entründung dex inneren Genitalien diesen Effect haben könnten, konnten nicht nachgewiesen werden; ebenso war auch bei den meisten Uaverheiratheten die Reizung durch Cohabitation und Infection mit Trippersecret ausgeschlossen. Catarrhe waren zwar öfters vorhanden, aber nicht so hochgradig und so lange dauernd, dass darauf die Anteflexion zurïckgefiiht werdeu konnte. Was ferner die Reizung der Douglas'sehen Falten durch zurückgehaltene harte Kothmassen anlangt, so kann dieses Moment kaum geltend gemacht werden. Ist doch Stuhlverstopfung eine fast constante Begleiterin der verschiedenartigen Uterinkrankheiten; so dass hier wohl die Vermuthung nabe liegt, es sei Ursache und Wirkung mit einander verwechselt worden. Wenn ferner Schultze in Betreff der verheiratheten Frauen von den "Thatsachen" spricht, dass die zu tiefe Immissio penis Entzündung und Schrumpfung der fraglichen Ligamenta herbeigefubrt habe, so müssen doch meiner Ansicht nach diese "Thatsachen" mit grosser Vorsicht verwertbet werden. Warum soll bei einer Fran, die in ihrem ledigen Stande an Dysmenorrhoe litt, in der Whe dann Jahre lang steril bleibt, und bei der Untersuchung eine Verkürzung der betreffenden Bünder zeigt, die Anteflexion nicht schon längst bestanden haben, die Affection der Bänder aber erst später hinzugetreten sein? Wäre der Uterus normal, so würde bald Schwangerschaft eintreten und hierdurch sowie durch die Geburt für die künftige Zeit eine schlaffe, weniger: vulnerable Vagina geschaffen worden sein. Tritt aber keine Conception ein, so werden die Cohabitationen bei der relativ straffen Beschaffenheit der Vagina fortgesetzt und diese Insulte können dann bei schon vorhandener Flexion an para- und perimetrisehen Exsudationen und ihren Folgen führen. In den sehr wenigen Fallen von Sterilität, wo Spuren von Verdickung des hinteren Gewölbes vorhanden waren, und wo tiber vage Schmerzen im Sbdomen auch ausserbalb der. Menstruation geklagt whurde, war mir diese Aufeinanderfolge viel wahrscheinlicher, als der von $\$ \mathrm{chultze}$ vermuthete Hergang.

Gehen wir nun zu den anatomischen Befunden und Symptomen über, durch welche sich die eigenartige Parametritis'posterior kundgeben soll. B. Schultze fuhrt hier die Starheit, die of nachweisbare Verdickung und die im Beginne der Erkrankung nie fehlende Empfindliehkeit der Douglas"sehen Falten sn. Wie man sieht, kön- 
nen die beiden letzten Zeichen, Verdickung und Empfindlichkeit fehlen, und es bleibt nur noch die Straffheit der Ligamenta übrig. Was die nur in frischen Fällen vorhanden sein sollende Empfindlichkeit anlangt, so möchte ich doch darauf aufmerksam machen, wie schmerzhaft oft bei Frauen, die nicht geboren haben, und bei jungfräulichen Individuen die manuelle Untersuchung überhaupt ist, einerlei ob man durch die Scheide oder das Rectum untersucht; meist steigert sich der Schmerz, wenn man eine so hochliegende Partie wie das hintere Scheidengewölbe austasten will. Da ist es denn oft schwer, eine bestimmte Stelle als Sitz des Schmerzes zu bezeichnen. Gesetzt den Fall auch, das hintere Gewölbe wäre bei der Berïhrung empfindlich, so dass also der Schmerz als von hier ausgehend angenommen werden muss, so ist es doeh nicht leicht zu sagen, dass gerade die fraglichen Ligamenta schmerzhaft seien. Geringe Exsudate und Extravasate im Douglas'sehen Raume selbst, dislocirte und fixirte Ovarien bedingen ebenfalls eine grosse Empindlichkeit. Dürften hier bei dem engen und beschränkten Terrain nicht leicht Verwechselungen vorkommen? Es lässt sich iabrigens aus den Schultze' schen Publicationen nicht entnehmen, ob er diese Empfindlichkeit constatirt hat bei seinem Manöver, durch Vorwärtsdrängen des Uterus die Constanz der Flexion zu prüfen; sollte dies der Fall sein, so sei nur bemerkt, dass bei Versuchen, den Uterus aus seiner Lage nach vorn zu drängen, durch Zerrung des hinteren Gewölbes auch ohne Affection der Ligamenta häufig Schmerzen hervorgerufen werden. In ähnlicher Weise verhält es sich mit der nach B. Schultze oft vorhandenen Verdickung der Ligamenta. Sehr häufig ist bei Nulliparen mit Anteflexion gar nichts nachzuweisen; oft nur eine geringe Resistenz im Seheidengewölbe zu constatiren; die verdickte Stelle ist dann oft von so unbestimmter Lage und Form, dass man katum sagen kann, ob Theile innerhalb oder ausserhalb des Peritoneums und welche zu dieser Erscheinung beigetragen haben. Nur selten und fast nur bei Frauen, die geboren haben, lässt sich aus der Lage, Richtung und Form der verdickten Partie eine Schwellung der Ligamenta utero-sacralia annehmen; fast immer konnte ich dieselbe auf abgelaufene puerperale Processe zurückführen.

Was nun die abnorme Straffheit der Ligamenta anlangt, auf welche Schultze in einer grossen Anzahl von Fällen einzig und allein seine Diagnose einer Parametritis posterior baut, so war es mir nur möglich, eine solche Beschaffenheit der Ligamenta zu constatiren unter zwei Bedingungen: 1) wenn dieselben sehr verdickt waren, oder 2) künstlich gespannt wurden. Ueber die erste Bedingung habe ich mich schon ausgesprochen; was die zweite betrifft, so werde ich gleich zeigen, wie wenig beweiskräftig derartige Fälle sind.

Ich komme nun zur Hauptsache: Wenn, wie B. Schultze glaubt, in der Mehrzahl der Fälle die hintere Fixation die Ursache der Anteflexion ist, so müssten doch nicht blos die verkürzten Liga. menta, sondern auch die Dislocation des Uterus selbst sowie dessen relative Unbeweglichkeit nachgewiesen werden können. Eine solche 
Rückwärtsstellung und Fixirung des Cervix durch den Finger festzustellen, ist ja nicht schwer; nach puerperalen Entzündungen hat man ja, wie bereits erwähnt, manchmal Gelegenheit, dieselbe freilich oft ohne Formveränderung des Uterus zu constatiren. Trotzdem findet man den bochgradig anteflectirten Uterus, der nach Schultze alle Symptome einer pathologischen Vorwärtsbeugung zeigt, nicht blos in der Mitte des Beskens stehend, sondern auch sehr beweglich; in manchen Fällen ist derselbe sogar mobiler als der normale Uterus. Freilich steht in vielen - aber nicht in allen - Fallen die Vaginalportion resp. ihre Spitze ausserhalb der Führungslinie des Beckens, sie ist nach hinten abgewichen, was aber leicht erklärbar ist, da ja viele Anteflexionen auch mit einer Anteversion verbunden sind; aber der obere Theil des Cervix, wo sich die Ligamenta sacro-uterina ansetzen, liegt ganz in der Mitte des Beckens. Führt man die Sonde ein und sucht man mit derselben den Uterus der Symphyse zu nähern, so kann man denselben bis auf $2-3 \mathrm{Cm}$. der vorderen Beckenwand nahe bringen, was sicher unmöglich wäre, wenn die straffer verKkïrzten Ligamenta den Uterus an der hinteren Beckenwand festhalten würden. Eine solche abnorme Fixation würde auch eine Verschiebung in der Richtung der Längsachse des Beckens, also in der Richtung nach oben erschweren; allein auch in dieser Richtung ist die Beweglichkeit des Uterus nicht gehindert, sondern, was man ebenfalls bei der Sondenuntersuchung constatiren kann, sogar noch vermehrt.

Vielleicht dürfte Schultze erwidern, die Faille, die ich hier schildere und im Auge habe, seien teine, pathologischen Flexionen", sondern nur "normale Vorwärtsbeugungen". Ich will hier nicht mit Schultze dariber rechten, wo die Grenzlinie zwischen diesen beiden Arten zu ziehen sei; ich werde daranf gleich zurỉekkommen. Aber gewiss wird er mir zugeben, dass in Fällen, wo der Winkel zwischen. Körper und Cervix ein sehr kleiner" und spitzer ist, ja manchmal bei einer vollständigen Retroversion des Cervix der anteHectirte Uteruskörper einfach auf der vorderen Fläche des Mutterbalses aufliegt, wo die Sonde auch mit zu Hülfenahme eines auf das vordere Scheidengewölbe ausgeäbten mannellen Druckes nur nach oft langem Bemühen äusserst schwer and mit Schmerzen ïber den inneren Muttermund des normal grossen Uterus vordringt, wo die Zeichen der sogenannten mechanischen Dysmenorrhoe exquisit ausgesprochen, wo sterilität vorhanden, dabei aber der Lterus ganz mobil ist, ich meine, dass es in diesen Fallen gewiss nicht der fast unausführbaren Untersuchung bei voller Blase oder des Druckes auf die hintere Fläche des Cervix bedarf, um die pathologische Natur dieser Formveränderung festzustellen.

Mit Recht dürfte aber doch Jemand fragen, wie es möglich sel, dass Schultze bei Anteflexionen so äusserst bäufig die fraglichen Veränderungen an den Donglas'schen Falten constatiren konnte, während ich nur in seltenen Fallen eine solche Dislocation des Cervix entdecken kann. Tch glaube, dieser Widersprach klïrt sich auf, wenn 
man die Art berücksichtigt, wie Schultze beim Nachweise seiner pathologisehen Flexion vorgeht. Er iibt zu diesem Zweeke entweder von der Scheide oder vom Mastdarme aus einen Druck "auf die hintere Fläche des Cervix. Hierbei müssen freilich dem untersuchenden Finger Bilder entstehen, die wir in der Schultze'schen Schilderung wieder erkennen. Bekanntlich gestatten die Uterusligamente der Gebärmutter eine ziemlich grosse Beweglichkeit; dies ist nur dadurch möglich, dass die Bandmassen für gewöhnlich nicht sehr gespannt sind; gespannt werden letztere erst dann, wenn die Bewegung in einer Richtung hin eine sehr excesșive wird. So verhält es sich auch mit den Ligamenta utero-sacralia, welche in den Douglas'schen Falten verlaufen. Untersucht man, ohne die Lage des Uterus zu ändern, so wird eine genaue Betastung des hinteren Gewölbes keine Ligamenta nachweisen können; wird aber der Uterus nach vorn und unten gedrängt, wie dies bei dem Schultze'schen Manöver der Fall ist, so müssen sich die Bänder spannen: die pathologische Straffheit der Ligamenta ist fertig. Ich habe dieses Experiment oft gemacht; nachdem ich die Sonde eingeführt, drängte ich den Uterus der vorderen Beckenwand zu; zuerst fühlt in dem hinteren Scheidengewölbe der untersuchende Finger eine undeutliche Resistenz; bei stärkerer Dislocation nach vorn können dann die Ligamenta als mehr oder weniger deutlich gespannte Stränge nachgewiesen werden. Bei Nachlass des Druckes nach vorn verschwindet auch wieder diese Erscheinung. So halte ich es für möglich, dass Schultze die normalen aber künstlich gespannten Ligamenta für pathologisch veränderte halten konnte.

Wie eben aus einander gesetzt, bin ich auf Grund meiner Erfahfahrungen und Untersuchungen ausser Stande, der Ansicht $B$. Schultze's, der zufolge die grosse Mehrzahl der Flexionen auf eine bestehende oder abgelaufene eigenthümliche Parametritis posterior zurückzuführen sei, besonders was die Nulliparen anlangt, beizupflichten. Diese Theorie bekundet meiner Ansicht nach einen wesentlichen Rückschritt gegenüber E. Martin, der die Verschiedenheit der ätiologischen Momente beim Zustandekommen der Anteflexionen betont. Zwar erwähnt auch B. Schultze noch eine Reihe anderer Entstehungsweisen, wie sie auch von Anderen angenommen werden; Alle sind aber nach seiner Ansicht gegenaber der hinteren Fixation des Uterus, recht seltene Befunde". Mit Befremden vermisst man unter dieser Kategorie die sogenannten angeborenen Flexionen, die, weil sie mit Dysmenorrhoe und Sterilität verbunden, von vielen Gynäkologen als pathologisch, und weil sie schon in der Pubertätszeit nachgewiesen werden können, als auf einen Entwickelungsfehler beruhend angesehen werden. Doch auch diese sind in der Arbeit von B. Schultze nicht ganz vergessen; nur werden dieselben nicht als pathologische betrachtet, sondern den normalen Flexionen zugezählt. ${ }^{1}$ ) Da aber in vielen solchen Fällen die Geschlechts-

1) Viel schwieriger als bei anderen Organen des Körpers ist die normale Lage und Form des Uterus am Iebenden Menschen festzustellen. Man kommt 
functionea nicht in regelmässiger Weise vor sich gehen, sondern die nämlichen Erscheinungen eintreten, wie bei den ,pathologischen Flexionen", so schreibt B. Schultze ganz consequent diese Symptome natiirlich nicht der Flexion an nnd für sich zu, sonderm es müssen Complicationen wie kindliche Kleinheit des Uterus, kindliche Enge des Cervix, Endometritis catarrhalis, mangelhafte Entwickelung der Ovarien zar Erklärung der Symptome herhalten. Die Complicationen sind die Hauptsache, die Anteflexion hat nur geringe oder gar keine Bedeutung. Auch diese Behauptung Schultae's scheint mir so wenig zutreffend, dass ich auf die von Letzterem aufgestellte Unterscheidung zwischen pathologischen und normalen Flexionen etwas näher eingehen muss.

Nach Schultze besteht der wesentliche Unterschied zwischen beiden nicht in der Grösse des Winkels oder der Symptome, sondern in einer Verminderung oder Aufhebung der normalen Biegsamkeit des Uterus. Diese, anomale Starrheit des Uterus in Anteflexion" wird aber constatirt - abgesehen von der abnormen Anheftung, die wir bereits besprochen - durch den Umstand, dass die gefuillte Blase den Uteruskörper nicht hebt, und dass durch einen Druck, ausgevibt mit dem Finger auf die hintere Fläche des Cervix, verbunden mit einem weiteren von den Bauchdecken aus iiber der symphyse auf das Corpus uteri ausgeibten Druck, keine Aufrichtung des Uteruskörpers erfolgt. Prüft man diejenigen Fälle, welche Schultze noch zu den normalen Flexionen rechnet, und bei denen er die begleitenden Affectionen als Lrsache der. Störangen ansieht, näher, so findet man, dass die Mehrzahl dass von ihm angegebene Kriterium der Normalität nicht aushält. Von einer manuellen Aufrichtung durch den Druck von hinten ist fast nie die Rede, der Uterus bleibt, auch wenn man noch über der Symphyse gegen den Körper drückt, anteflectirt. Was die Aufrichtung durch die Füllung der Blase anlangt, so ist, wie Schultze selbst sugiebt, die Controle der Aufrichtung sehr schwierig. Gewiss wird mir aber Schultzo zugestehen, dass in all' den Fallen, in denen dio Blase oder ein Druck vou hinten den Uterus zu heben vermag, dies auch mit Leiohtigkeit durch die Sonde, verbunden mit einem auf das vordere Scheidengewölbe ausgeibbten Druck geschehen kann. Aber wie unendlich schwierig, ja manchmal unmöglich, ist selbst ftir den Geüibteren die Geradestreckung des von Schultze noch zu den normal Flectirten gerechneten Uterus! Soll eine solche Beugung nogh eine normale genannt werden können?

ja - um nur auf einen Punkt hinzuw eiken - so selten in die ILage, an einem geschlechtsgesunden Woibe eine Untersuchung der inneren Genitalien roramehmen. Wir sind also auf die Untersuchung von geschlechtakranken Individuen angewiesen. Das grösste Contingent dex gynäkologischen Erkrankungen stellt aber der Uterus; also ein unbrathohares Material. Aber anch die Falle von Erkrankung anderer Geschlechtsthelle lassen sich bei dem innigen Zusammonhange der Genewationsorgane unter einduder nicht ohne Bedenken zu fraglichem $Z$ wecke verwerthen. 
Gehen wir schliesslich zu den Affectionen über, die bei normal anteflectirten nicht fixirten Uterus manchmal dysmenorrhoische oder ähnliche Erscheinungen bewirken sollen. Schultze führt zuerst die Kleinheit des Uterus an. Allerdings giebt es Fälle, wo der kleine, walzenförmige, dünnwandige Uterus in Anteflexionsstellung angetroffen wird, wo dann auch die charakteristischen Symptome der Formveränderung fehlen - meist bei chlorotisch amenorrhoisehen Individuen - aber in der Mehrzahl der Fälle, wo auch die Erscheinungen der Dysmenorrhoea ausgesprochen sind, zeigt der Uterus nach Einführung der Sonde nicht blos die normale Länge, sondern auch die regelrechte Dicke der Wandung; auch die stärkere Entwickelung des Uteruskörpers gegenüber dem Cervix war ausgesprochen - kurz, es entsprach der Uterus ganz dem Alter der Patientin. Man erhält in solchen Fällen ganz den Eindruck, als hätte sich die Gebärmutter regelmässig entwickelt, nur wäre die durch die Vergrösserung des Uteruskörpers gewöhnlich bewirkte Aufrichtung und Streckung ausgeblieben. Dass nicht die kindliche Enge des Cervicalkanales die Störung bewirkt, sondern die Knickung selbst, kann ja in solchen Fällen dadureh nachgewiesen werden, dass die Sonde ohne allen Anstand durch den äusseren Muttermund und durch den Cervicalkanal bis zum Knickungswinkel vordringt, hier auf einen bedeutenden schwer zu überwindenden Widerstand stösst, dass aber nach Aufrichtung und Fixirung des Uterus in seiner normalen Lage und Form die Sonde kein Hinderniss am Os internum findet. Was nun den Catarrh anlangt, so will ich gern zugeben, dass derselbe nicht blos sehr häufig vorhanden, sondern auch Menstruationsbeschwerden vielleicht weniger hervorrufen, als dieselben vermehren kann; aber entschieden ist derselbe trotz Dysmenorrhoe in der Mehrzahl der Fälle, die frühzeitig nach Eintritt der Menstruationen zur Beobachtung kommen, nicht vorhanden; meist tritt er ja erst später auf. Wenn von B. Schultze auch die „Ovarian pain" beigezogen wird, um die Dysmenorrhoe der angeborenen Flexionen zu erklären, so soll ihm hier insofern nicht widersprochen werden, als oft der Charakter der Schmerzen nicht deutlich ausgeprägt ist; aber nur zu häufig sind die Schilderungen der Schmerzensempfindung so klar, dass die Uterinalkoliken als Folge der mechanischen Störungen im Uterus durchaus nicht verkannt werden können. So lässt sich bei einer grossen Anzahl von Fällen nachweisen, dass das Primäre der Störungen in der Flexion zu suchen sei. Letztere bedingt die dysmenorrhoischen Erscheinungen; nachdem dieselben eine Zeit lang gedauert haben', tritt sehr oft der Genitalkatarrh auf und später, wenn auch nicht so häufig, kommt es zur chronischen Metritis, wozu sich dann noch Affectionen in den Nachbartheilen hinzugesellen können.

Soweit meine Erfahrungen. Fasse ich das Gesagte zusammen, so ergeben sich Sätze, die mit der Schultze'schen Annahme sehr wenig harmoniren:

1) Bei Nulliparen mit Anteflexionen ist der vorwärts gebeugte Uterus meist frei beweglich, die hintere Fixation durch Schrumpfung 
der Ligamenta sacro-uterina abex kein häufiger Befund. Wo letztere. angetroffen wird, ist es oft zweifelhaft, ob die Veränderung der Bänder nicht erst nachträglich entstanden.

2) Die meisten Anteflexionen ohne Fixirung an der hinteren Beckenwand bei Nulliparen diurfen nicht den normalen, sondern den pathologisehen Beugungen zugesellt werden; die Störungen der Geschlechtsfunctionen sind meist auf die Flexion selbst, seltener auf Complicationen zurückzuftibren.

\title{
Ueber Cysten des breiten Mutterbandes.
}

\author{
Nachtrag za Band IX dieses Archivs, S. 478. \\ Vor
}

A. Gusserow.

Win eigeuthümlicher Zufall hat es gefügt, dass, als der Druck der Mittheilung im IX. Bande dieses Archivs vollendet war, die betreffende Kranke sich wieder in meiner Klinik einfand. Während dieselbe noch im Februar dieses Jahres geschrieben hatte, dass ihr Befinden ein gutes sei, hatte sie gleichzeitig verschwiegen, das wiederum eine Zunahme des Abdomen einzutreten schien. Von Ende Februar war nun dieses Wachsthum der Geschwulst so bedeutend, dass am 30. Mai der grösste Umfang des Leibes $90 \mathrm{Cm}$. betrug, über dem Nabel gemessen 87. Die Entfernung von der Symphyse aum höehsten Punkte des Tumor betrug 31, die Entfernung von einer Spina anterior zur anderen $41 \mathrm{Cm}$.

Im Uebrigen waren alle Erscheinungen seitens der Geschwulst (Fluctuation, Dämpfing, Verhalten zum Uterus) ganz die gleiche wie im October 1874 - es handelte sich einfach tim eine Wiederfüllung der Cyste bei vollkornmen ungestörtem Wohlbefinden der Kranken.

Es bedarf keiner weiteren Auseinandersetzungen, dass nun sofort die Exstirpation der Geschwulst indicirt war. Die Incision wurde in Voraussicht der einfächerigen Beschaffenheit des Tumors so klein wie möglich gemacht und betrug $6-8 \mathrm{Cm}$. Die Operation verlief ohne alle bemerkenswerthe Zwischenfallle. Zwei Adhäsionen mit dem Omentum mussten doppelt unterbunden und durchschnitten werden. Die Geschwulst entleerte 6200 Gramm einer vollkommen wasserhellen Flisssigkeit, die ganz dieselbe Beschaffenheit hatte, wie die im No- 